\title{
Structure Design of Micromechanical Silicon Resonant Accelerometer
}

\author{
Libin Huang*, Weiwei Chen, Yunfang Ni, Yang Gao and Liye Zhao \\ School of Instrument Science and Engineering, Southeast University, Nanjing 210096, China \\ Key Laboratory of Micro-Inertial Instrument and Advanced Navigation Technology, \\ Ministry of Education
}

(Received October 15, 2012; accepted December 27, 2012)

Key words: accelerometer, resonator, structure design, finite element analysis

The micromechanical silicon resonant accelerometer is one of the development directions toward high-performance micromechanical accelerometers. We designed a differential micromechanical silicon resonant accelerometer based on double-ended tuning fork (DETF) resonators and single-stage microleverage mechanisms. The effect of the gap on the resonance characteristic of the resonant beams in the DETF structure was investigated through modal analysis and harmonic response analysis. An improved microleverage mechanism was designed. Compared with the conventional mechanism, this microleverage mechanism can markedly improve the sensitivity of the accelerometer. The overall structure of the accelerometer was designed in combination with the present microfabrication technology. The simulation of the overall structure was carried out using ANSYS software. Then the accelerometer was fabricated through the silicon on glass (SOG) process and packaged in a ceramic shell. Test results show that the unloaded resonant frequencies of the packaged accelerometer resonators are 30.519 and $30.448 \mathrm{kHz}$. The quality factor is 335 and the scale factor is $84.52 \mathrm{~Hz} / \mathrm{g}$. The test results prove the feasibility of the accelerometer design. This study lays a good foundation for subsequent development work.

\section{Introduction}

At present, most micromechanical silicon accelerometers adopt capacitance detection methods, which have the advantages of small temperature drift, high sensitivity, good reliability, and good stability. However, the structure size of the micromechanical silicon accelerometer is greatly narrowed, markedly reducing the sensitivity and resolution of the instrument. Moreover, the capacitance detection method is strongly affected by the parasitic effect and the noise has basically reached the detection limit, making it difficult to further improve the measurement accuracy significantly. ${ }^{(1)}$

The basic characteristic of the micromechanical silicon resonant accelerometer is

${ }^{*}$ Corresponding author: e-mail: huanglibin@seu.edu.cn 
its digital output signal, which has the advantages of easy detection, good antijamming capability, and smaller errors in transmission and processing. Therefore, this type of sensor relatively easily realizes high-precision measurement, thus becoming one of the development directions toward high-performance micromechanical accelerometers. ${ }^{(2)}$ Many famous research institutes, both domestic and overseas, have carried out research on this type of accelerometer. Draper Laboratory is one of the pioneers of the study of micromechanical silicon resonant accelerometers, and their results are still at the cutting edge of international research. The principle prototype it developed provides the best overall performance, with a scale factor stability better than $1 \mathrm{ppm}$ and a bias stability superior to $1 \mu \mathrm{g} .^{(3)}$ Researchers at the University of California, Berkeley, have focused on the study of the micromechanical leverage mechanism; ${ }^{(4,5)}$ Researchers at Seoul National University in Korea have published a number of research findings on vacuum packaging, closed-loop stability, and the effect of temperature; ${ }^{(6,7)}$ Temic Telefunken Microelectronic and the Mercedes-Benz Technology Center in Germany and the University of Toronto in Canada have analyzed the resonance characteristic of resonant beams, based on which detailed requirements were proposed for the structure parameters of resonant beams and the positions of drive combs. ${ }^{\left({ }^{(8,9)}\right.}$ Researchers at the University of Southampton in the UK have discussed the structure design, theoretical calculation, and processing technology of micromechanical silicon double-ended tuning fork (DETF) resonators. ${ }^{(10,11)}$ The major domestic research institutes are Tsinghua University, Peking University, Tianjin University, Nanjing University of Science and Technology, Southeast University, North University of China, Chongqing University, and China Academy of Engineering Physics. Their main research topics include the structure design of micromechanical silicon resonant accelerometers, nonlinear effects, microfabrication technology, frequency detection methods, and laboratory principle prototypes..$^{(2,12)}$

In related articles, the optimal design of the DETF resonator is discussed. However, the effect of the value of the gap between the two beams of the DETF has seldom been considered in depth. In this study, the effect of the gap on the resonance characteristic of the resonant beams was investigated through modal analysis and harmonic response analysis. An improved microleverage mechanism was designed to increase the sensitivity of the accelerometer. Then, on this basis, the design of a micromechanical silicon resonant accelerometer with DETF resonators and single-stage microleverage mechanisms was accomplished.

\section{Structure Design}

\subsection{Working principle of the micromechanical silicon resonant accelerometer}

The micromechanical silicon resonant accelerometer is composed of a proof mass, several suspension beams, microleverage mechanisms, and resonators. A schematic diagram of the structure is shown in Fig. 1. The two resonators have the same size and are symmetrically arranged through the center of the mass. When accelerated, the proof mass generates axial forces on the two resonators, which change their resonant frequencies. This frequency change is measured and serves as the indicated output of the sensor. The two resonators are configured in such a way that the axial forces have 


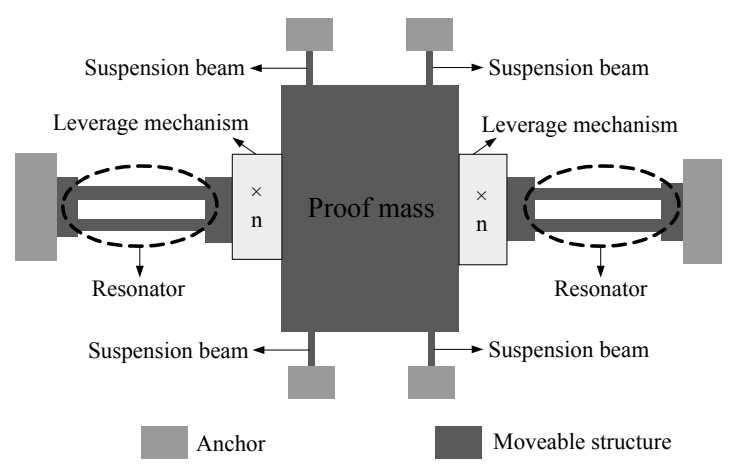

Fig. 1. Schematic diagram of the micromechanical silicon resonant accelerometer.

different effects, that is, when one resonator is subjected to tension and its resonant frequency increases, the other one is compressed and its resonant frequency decreases. The value of acceleration can be calculated from the difference in the frequencies. ${ }^{(3)}$ This differential design doubles the sensitivity of the accelerometer and eliminates commonmode errors.

\subsection{Optimal design of the resonator}

The resonator design is a key component in the structure design of a resonant accelerometer because the resonator is closely linked to the sensitivity, linearity, range, and other parameters of the accelerometer.

With appropriate excitation, the two beams of the DETF can realize an antiphase vibration in the same plane. On this occasion, the sum of the forces and moments generated by the two beams is equal to zero at their fixed supports, hence the energy coupled from the resonator through its fixed supports to the surrounding environment can be reduced dramatically and a high Q-factor can be achieved for the resonator. Beeby and Tudor reported an improved DETF (as shown in Fig. 2) that can greatly reduce stress compared with the basic DETF. ${ }^{(13)}$ Their structure is used in our accelerometer.

Within the linear range, the frequency difference of the differential accelerometer is calculated using

$$
\Delta f=f_{0} \cdot \frac{A \rho A_{\mathrm{S}} a}{4} \cdot \frac{0.295 L^{2}}{E w^{3}}
$$

where $f_{0}$ is the nominal unloaded resonant frequency, $A$ is the amplification factor of the leverage mechanism, $A_{\mathrm{S}}$ is the surface area of the proof mass, $a$ is the acceleration, $\rho$ is the density of the material, $L$ is the length of the resonant beam, $w$ is the width of the resonant beam, and $E$ is the Young's modulus of the material. From eq. (1), it can be seen that, in order to improve the scale factor of the accelerometer, the resonant beam should be as long and narrow as possible. In accordance with the limitations of the process, the width of the resonant beam is set to $8 \mu \mathrm{m}$ and the length is set to $1000 \mu \mathrm{m}$. 


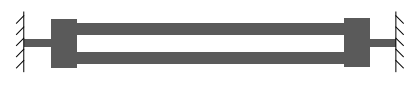

DETF+outriggers + stubs

Fig. 2. Schematic diagram of the improved DETF.

Table 1

Resonant frequency with different gap values.

\begin{tabular}{lrrrrr}
\hline Gap value $(\mu \mathrm{m})$ & 50 & \multicolumn{1}{c}{40} & \multicolumn{1}{c}{30} & \multicolumn{1}{c}{20} & \multicolumn{1}{c}{10} \\
\hline Frequency of first mode $(\mathrm{Hz})$ & 68416 & 67766 & 66673 & 64652 & 60789 \\
Frequency of second mode $(\mathrm{Hz})$ & 70019 & 70010 & 70007 & 69982 & 69978 \\
Difference between two frequencies $(\mathrm{Hz})$ & 1603 & 2244 & 3334 & 5330 & 9189 \\
\hline
\end{tabular}

The length of the stub is set to $30 \mu \mathrm{m}$ and the width is set to $8 \mu \mathrm{m}$.

The overall performance of the resonator is greatly affected by the value of the gap between the two beams of the DETF. To determine the appropriate gap, several modal analyses were carried out using ANSYS software. With other parameters at constant values, the gaps were taken as $10,20,30,40$, and $50 \mu \mathrm{m}$. The simulated results are shown in Table 1.

The first mode is an interference mode, in which the two beams exhibit an in-phase vibration. The second mode is the operation mode, in which the two beams exhibit an antiphase vibration. To obtain good performance, the frequency difference between these two modes should be large. From Table 1, it can be seen that, as the gap increases, the frequency difference decreases. Hence, to obtain a large frequency difference, a small gap is preferred.

Harmonic response analyses were also carried out. The driving force was applied to the left beam in these analyses. The amplitude-frequency responses of the two beams of the DETF are shown in Fig. 3.

Figure 3 shows that the amplitude-frequency response curve has two peaks, i.e, the first mode and second mode of the resonator. As the gap increases, the frequency difference between the two modes monotonically decreases. When the gap is relatively small, such as 10 or $20 \mu \mathrm{m}$, although the driving force is applied to one beam in these analyses, the other beam vibrates with the same amplitude and frequency at the same time. As the gap increases, the two beams vibrate in a relatively independent manner as shown in Figs. 3(g)-3(j). This shows that the mutual effect between the two resonant beams gradually decreases as the gap increases and that the nature of the DETF is gradually weakened.

To obtain the amplitude-frequency response curve of the DETF under normal working conditions, driving forces with equal value and opposite directions were applied to the two beams, making them vibrate in an antiphase manner. With no consideration of the processing error, the amplitude-frequency response curves of the two beams would have the same peak value, as shown in Fig. 4. For the present domestic microfabrication technology, a certain value of processing error of the beam width was considered. 


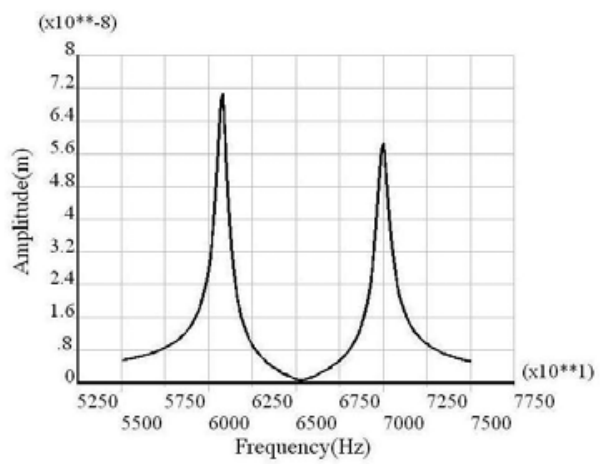

(a)

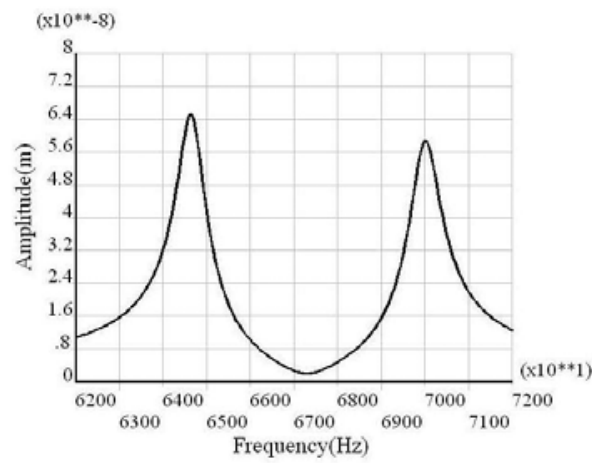

(c)

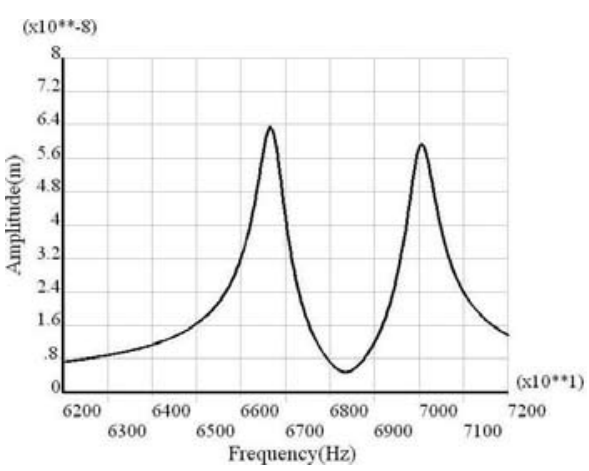

(e)

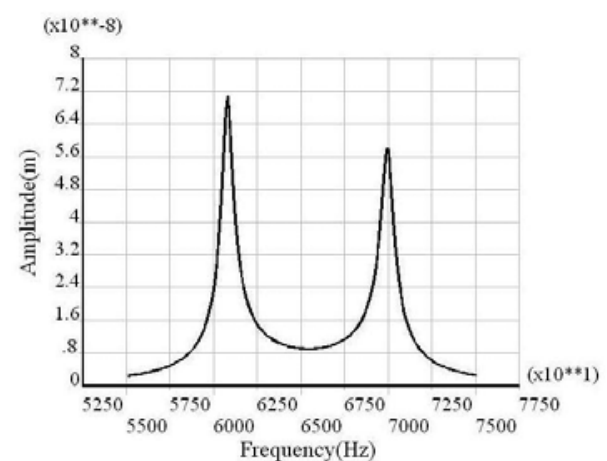

(b)

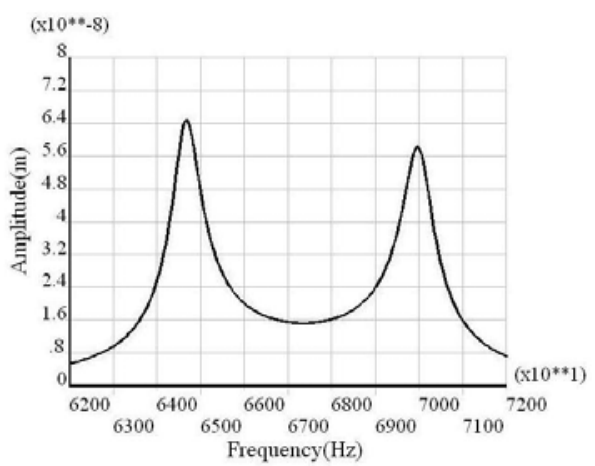

(d)

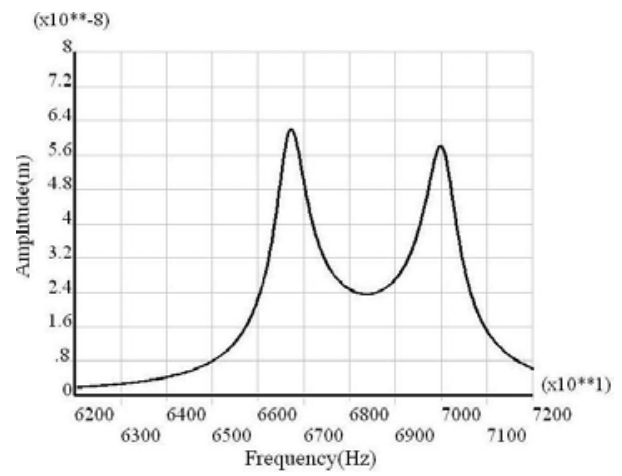

(f)

Fig. 3. Amplitude-frequency response curves (unilateral driving): (a) left beam (gap value: 10 $\mu \mathrm{m}$ ), (b) right beam (gap value: $10 \mu \mathrm{m}$ ), (c) left beam (gap value: $20 \mu \mathrm{m}$ ), (d) right beam (gap value: $20 \mu \mathrm{m}$ ), (e) left beam (gap value: $30 \mu \mathrm{m}$ ), (f) right beam (gap value: $30 \mu \mathrm{m}$ ), (g) left beam (gap value: $40 \mu \mathrm{m}$ ), (h) right beam (gap value: $40 \mu \mathrm{m}$ ), (i) left beam (gap value: $50 \mu \mathrm{m}$ ), and (j) right beam (gap value: $50 \mu \mathrm{m}$ ). 


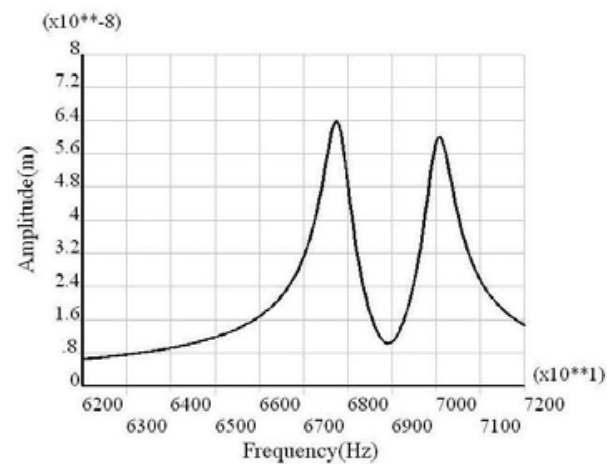

(g)

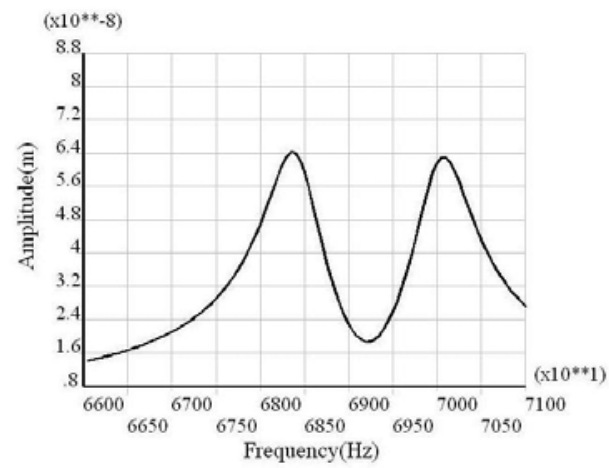

(i)

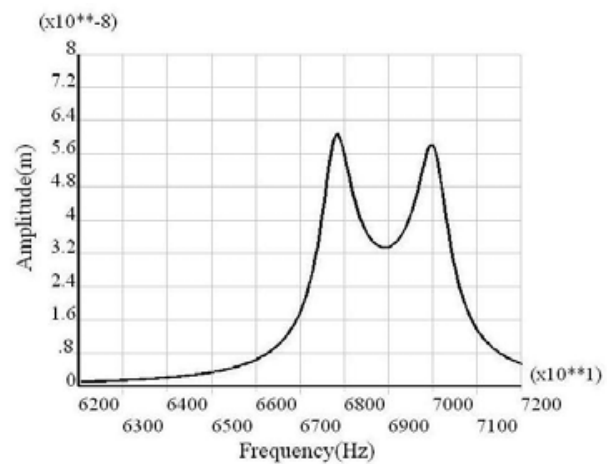

(h)

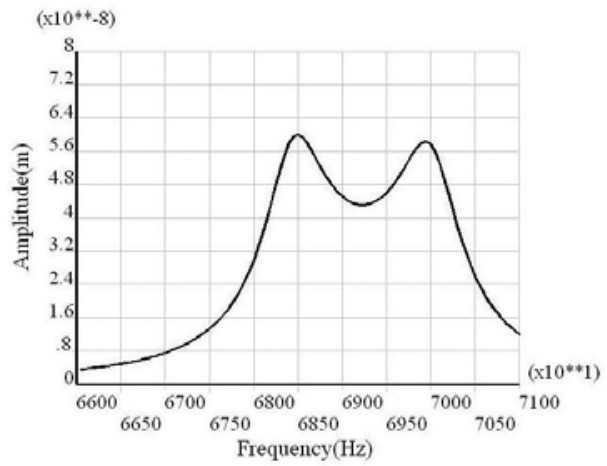

(j)

Fig. 3. (continued).

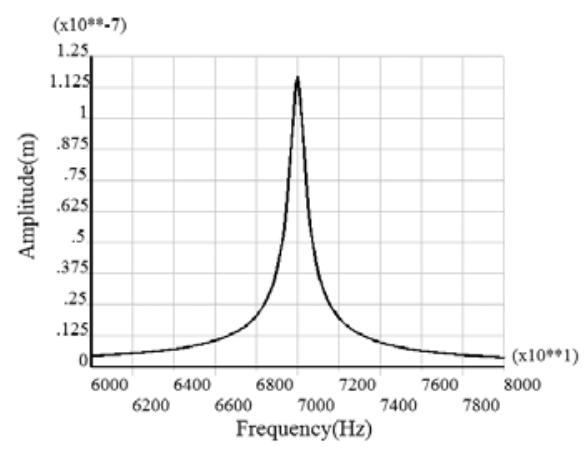

(a)

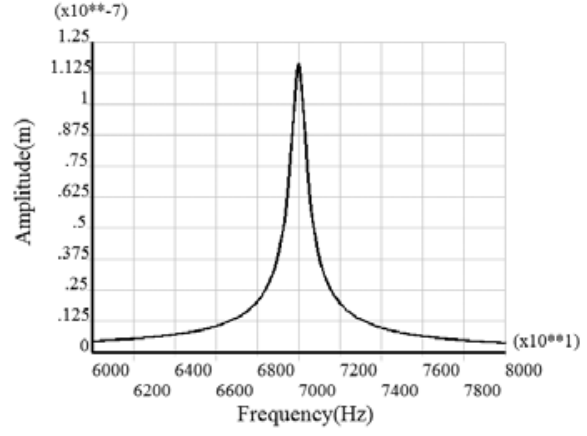

(b)

Fig. 4. Amplitude-frequency response curves (bilateral driving, gap value is $20 \mu \mathrm{m}$ ). 
Supposing the width of the left beam is $8 \mu \mathrm{m}$ and that of the right beam is $8.1 \mu \mathrm{m}$, harmonic response analyses were carried out on DETF resonators with different gaps. The resonant frequencies of the two beams were collected and are shown in Table 2.

From Table 2, it can be concluded that the difference between the resonant frequencies of the two beams increases as the gap increases for the same processing error. The above analysis results were obtained when the damping ratio was set as 0.005 . When the damping ratio was set smaller, the difference between the resonant frequencies of the two beams was decreased. For example, when the gap was $20 \mu \mathrm{m}$, reducing the damping ratio by an order of magnitude makes the difference between the resonant frequencies decrease from $3 \mathrm{~Hz}$ to less than $0.1 \mathrm{~Hz}$. Thus, it can be seen that a higherlevel vacuum package would be beneficial for decreasing the difference between the resonant frequencies of the two beams caused by a processing error.

In conclusion, when the gap of a DETF increases to a certain extent and the two beams have different widths, the DETF becomes equivalent to a simple combination of two resonant beams vibrating independently. In that case, the high Q-factor of the DETF resonator in theory would probably not be achieved.

Considering the above factors, the gap between the two resonant beams should not be too large. Nevertheless, when the gap is too small, the squeeze film damping induced between the two beams cannot be neglected. The coefficient of the damping force is given by ${ }^{(14)}$

$$
c_{\mathrm{b}}=\frac{\mu h^{3} L}{d_{\mathrm{b}}^{3}} \beta(\eta)
$$

where $\mu$ is the gas viscosity coefficient, $h$ is the thickness of the resonant beams, $d_{\mathrm{b}}$ is the gap between the two resonant beams, and $\beta(\eta)$ is a correction factor.

The calculation results show that when the gap is decreased from 20 to $10 \mu \mathrm{m}$, the coefficient of the damping force is increased by an order of magnitude. Taking into account the fact that the vacuum degree of the present accelerometer package is not sufficiently high, the final gap chosen in the design was $20 \mu \mathrm{m}$.

The final design of the resonator is shown in Fig. 5, and employs specific combs to realize electrostatic driving and capacitive frequency sensing. Figure 6 gives the simulated force-frequency sensitivity of the resonator obtained by prestress modal analysis. After fitting, the force-frequency sensitivity of the resonator is $0.43 \mathrm{~Hz} / \mu \mathrm{N}$.

Table 2

Resonant frequencies of the resonant beams.

\begin{tabular}{lrrrrr}
\hline Gap $(\mu \mathrm{m})$ & 50 & \multicolumn{1}{c}{40} & 30 & \multicolumn{1}{c}{20} & \multicolumn{1}{c}{10} \\
\hline Resonant frequency of left beam $(\mathrm{Hz})$ & 70618 & 70523 & 70497 & 70440 & 70629 \\
Resonant frequency of right beam $(\mathrm{Hz})$ & 70583 & 70505 & 70488 & 70437 & 70628 \\
Difference between two frequencies $(\mathrm{Hz})$ & 35 & 18 & 9 & 3 & 1 \\
\hline
\end{tabular}



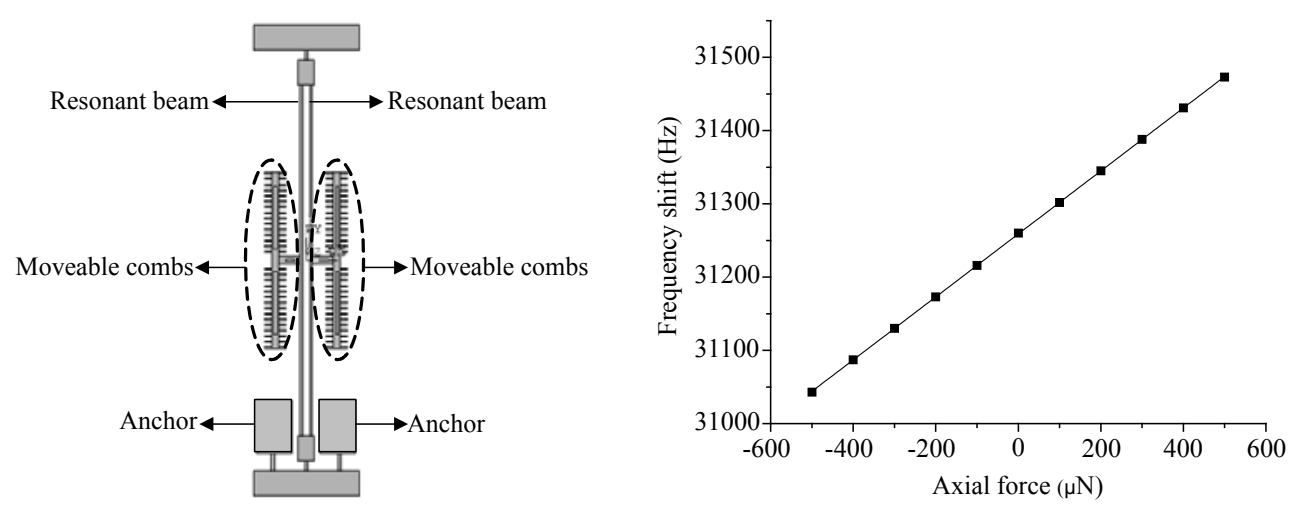

Fig. 5 (left). Schematic diagram of the resonator structure.

Fig. 6 (right). Simulated force-frequency sensitivity of the resonator.

\subsection{Design of the microleverage structure}

To obtain higher sensitivity, a single-stage microleverage mechanism is designed in the resonant accelerometer to amplify the inertial force generated by the proof mass.

Owing to the limitations of the processing conditions and the layout of the resonator, a pivot and anchor are placed on the same side of the leverage. The structure of a conventional microleverage mechanism is shown in Fig. 7(a), in which the input beam is parallel to the output beam; the improved structure is shown in Fig. 7(b), in which it can be seen that the input beam is perpendicular to the output beam. On the basis of previous studies, the relevant parameters were set as follows: the length of the input beam was set to $50 \mu \mathrm{m}$ and the width was set to $10 \mu \mathrm{m}$, the length of the output beam was set to 60 $\mu \mathrm{m}$ and the width was set to $10 \mu \mathrm{m}$, the length of the pivot beam was set to $80 \mu \mathrm{m}$ and the width was set to $10 \mu \mathrm{m}$, the length of the lever arm was set to $1150 \mu \mathrm{m}$ and the width was set to $70 \mu \mathrm{m}$. Schematic diagrams of the resonator with different microleverage mechanisms are shown in Fig. 8. Figure 9 gives the simulated force-frequency sensitivities of the resonator with different microleverage mechanisms obtained by prestress modal analysis. After fitting, the force-frequency sensitivities of the resonator with the conventional and improved microleverage mechanisms are 4.83 and 4.84 $\mathrm{Hz} / \mu \mathrm{N}$, respectively. The amplification factor of the conventional microleverage is 11.23 and that of the improved microleverage is 11.26. The amplications of the two microleverages are almost equal.

\subsection{Design of the suspension beam}

In micromechanical structure design, the shape of the suspension beams is a pivotal design element that affects the performance dramatically. At present, common suspension beams include straight beams, folded beams, L-shaped beams, fishhook beams, S-shaped beams, and inclined beams. The straight beam is simplest in structure. Nevertheless, it is susceptible to residual stress. The frequency difference between the 


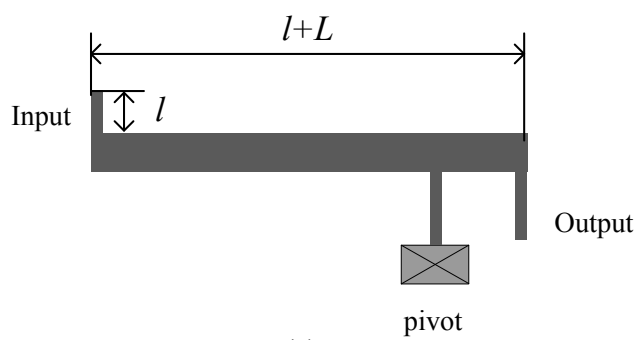

(a)

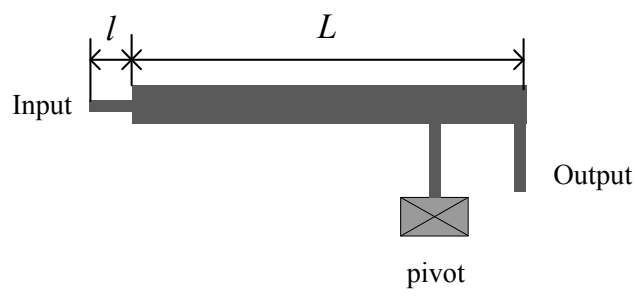

(b)

Fig. 7. Schematic diagrams of the microleverage structures: (a) conventional microleverage structure and (b) improved microleverage structure.

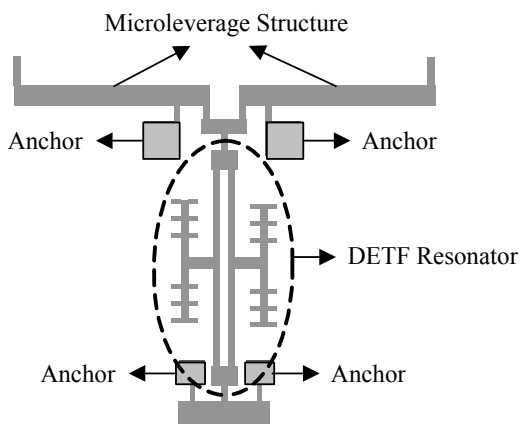

(a)

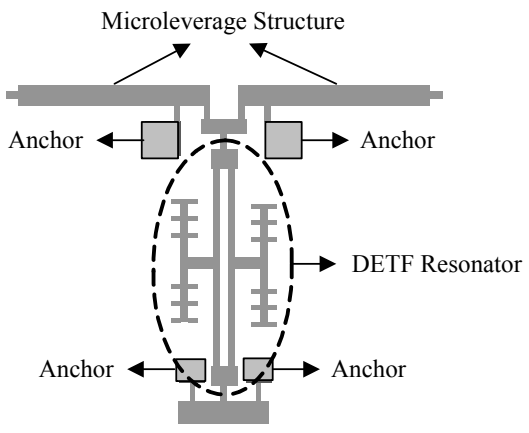

(b)

Fig. 8. Schematic diagrams of the resonator with the microleverage structures: (a) resonator with the conventional microleverage structure and (b) resonator with the improved microleverage structure.

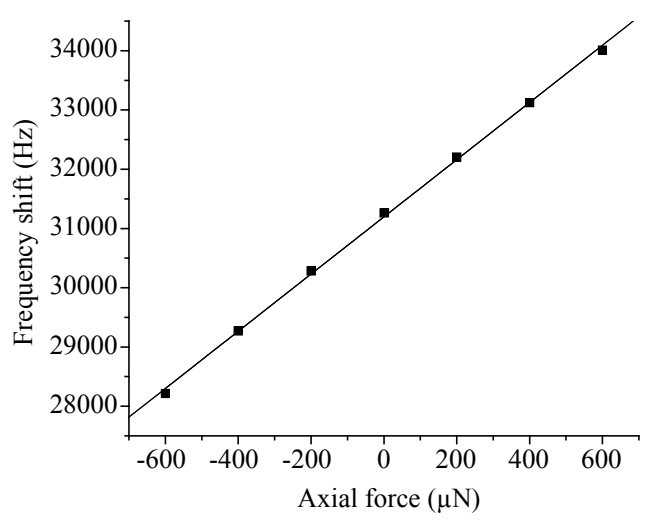

(a)

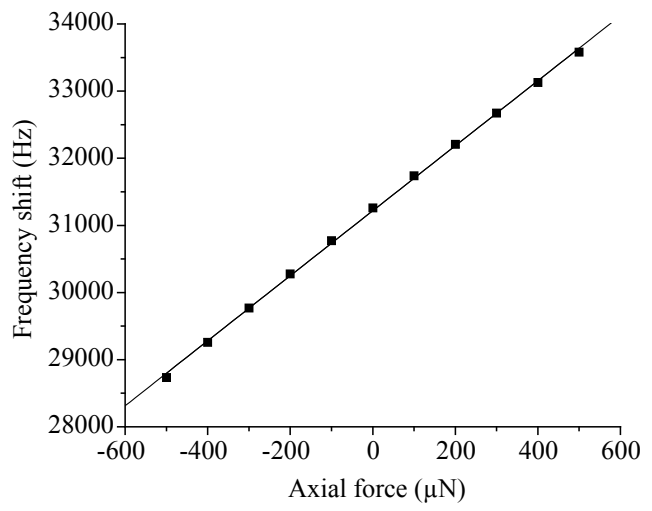

(b)

Fig. 9. Simulated force-frequency sensitivities of the resonator with the microleverage structures: (a) resonator with the conventional microleverage structure and (b) resonator with the improved microleverage structure. 
first two modes of L-shaped beams, fishhook beams, S-shaped beams, and inclined beams is very small. Hence, they are sensitive to cross-axis acceleration and are inappropriate for use in a single-axis accelerometer.

To obtain high resolution, low cross-axis sensitivity, and good antijamming capability, a single-axis accelerometer requires suspension beams with small stiffness in the sensing direction and large stiffness in other directions. On this basis, a folded beam would be most suitable. Moreover, a folded beam has a relatively simple structure, has stress release capability, and can resist the expansion caused by processing or other thermal changes. It also has easily adjustable stiffness and modes. Therefore, a folded beam was adopted in the design of the accelerometer.

\subsection{Overall structure of the accelerometer}

The micromechanical silicon resonant accelerometer mainly consists of a proof mass, two resonators, four microleverages, four suspension beams, and several anchors. The upper and lower resonators are completely symmetrically distributed. Two structures of the micromechanical silicon resonant accelerometer with different microleverages are shown in Fig. 10. The frequency differences of the two DETF resonators under different accelerations were obtained through ANSYS simulation, as shown in Fig. 11. After fitting, it was found that the scale factor of the accelerometer with the conventional microleverage is $42 \mathrm{~Hz} / \mathrm{g}$ and that of the accelerometer with the improved microleverage is $63 \mathrm{~Hz} / \mathrm{g}$. A comparison was carried out of the axial stress values at the same node of the upper DETF for the two accelerometer structures. The results are shown in Table 3. It is clear that the improved microleverage induces larger axial stress on the resonant

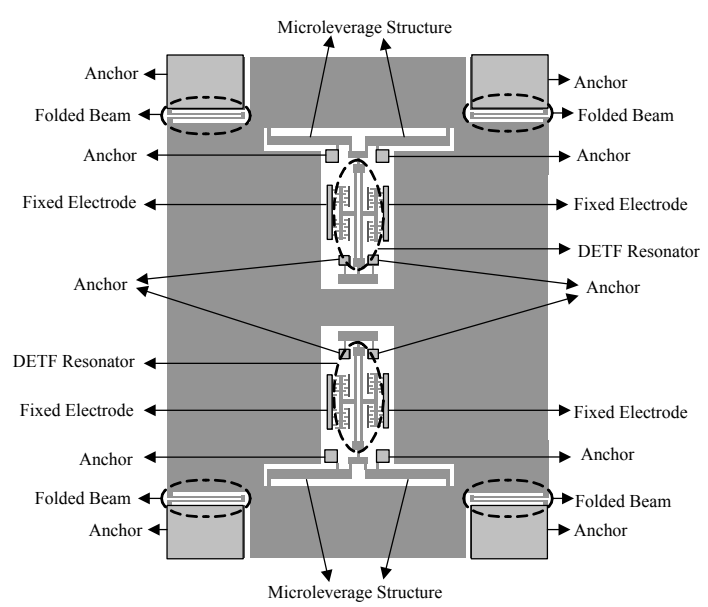

(a)

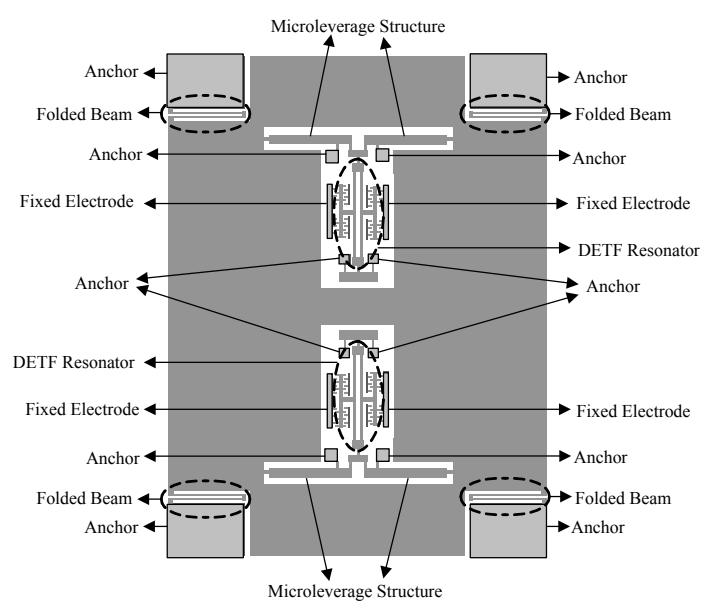

(b)

Fig. 10. Schematic diagrams of the accelerometer: (a) accelerometer using the conventional microleverage structure and (b) accelerometer using the improved microleverage structure. 


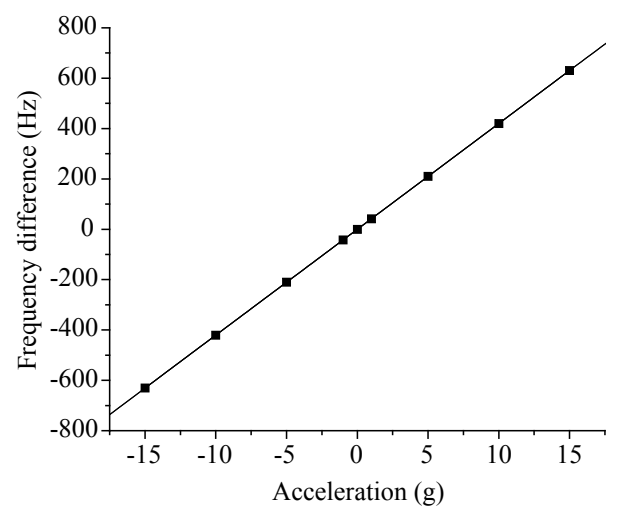

(a)

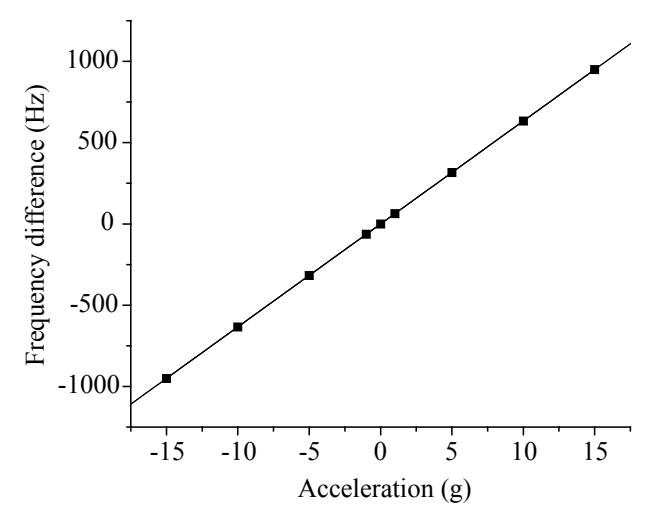

(b)

Fig. 11. Simulated scale factors of the accelerometer: (a) accelerometer using the conventional microleverage and (b) accelerometer using the improved microleverage.

Table 3

Comparison of axial stresses of the two accelerometers.

\begin{tabular}{lcccc}
\hline Acceleration & $1 g$ & $5 g$ & $10 g$ & $15 g$ \\
\hline Axial stress of accelerometer (a) $(\mathrm{MPa})$ & $0.027399 \mathrm{E}+6$ & $0.13700 \mathrm{E}+6$ & $0.27399 \mathrm{E}+6$ & $0.41099 \mathrm{E}+6$ \\
Axial stress of accelerometer (b) (MPa) & $0.041304 \mathrm{E}+6$ & $0.20652 \mathrm{E}+6$ & $0.41304 \mathrm{E}+6$ & $0.61957 \mathrm{E}+6$ \\
\hline
\end{tabular}

beams under the same acceleration, thus leading to a larger frequency change. It can be concluded that, even with the same amplification factor, microleverages with different mechanisms can result in different sensitivities of the accelerometers. The improved microleverage was adopted in the final design.

Figure 12(a) shows the working mode of the proof mass, Fig. 12(b) shows the working mode of the upper resonator, and Fig. 12(c) shows the working mode of the lower resonator. The unloaded resonant frequency of the resonator is $31.263 \mathrm{kHz}$.

\section{Fabrication and Package}

A prototype device was fabricated using the silicon on glass (SOG) process and the main process steps are illustrated in ref. 15. Figure 13 shows the resonator of the fabricated resonant accelerometer observed under a microscope.

The fabricated accelerometer was packaged in a ceramic shell, as shown in Fig. 14. It has an overall size of $10 \times 10 \times 4 \mathrm{~mm}^{3}$. Figure 15 shows the internal structure of the ceramic shell after wire bonding. 


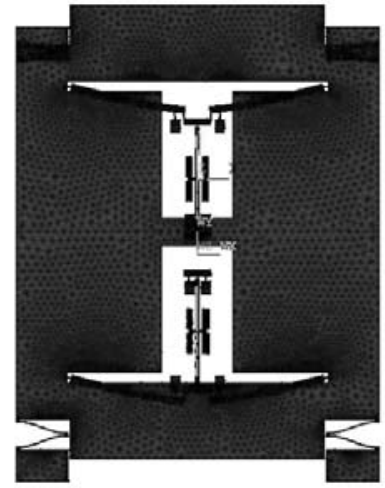

(a)

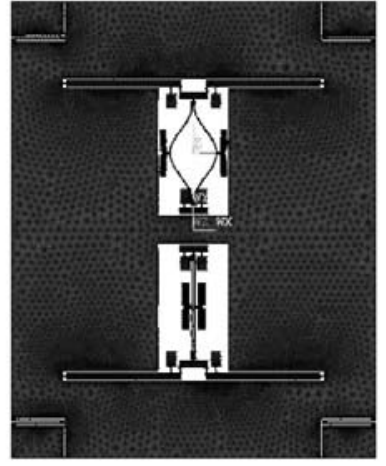

(b)

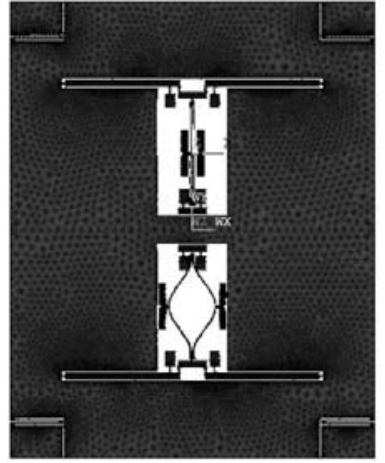

(c)

Fig. 12. Modes of the micromechanical silicon resonant accelerometer: (a) working mode of the proof mass, (b) working mode of the upper resonator, and (c) working mode of the lower resonator.

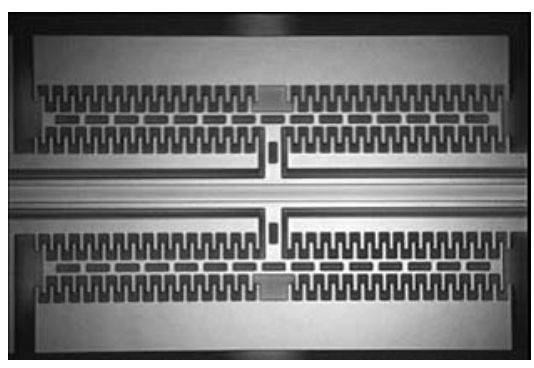

Fig. 13. Resonator of the fabricated resonant accelerometer observed under a microscope.
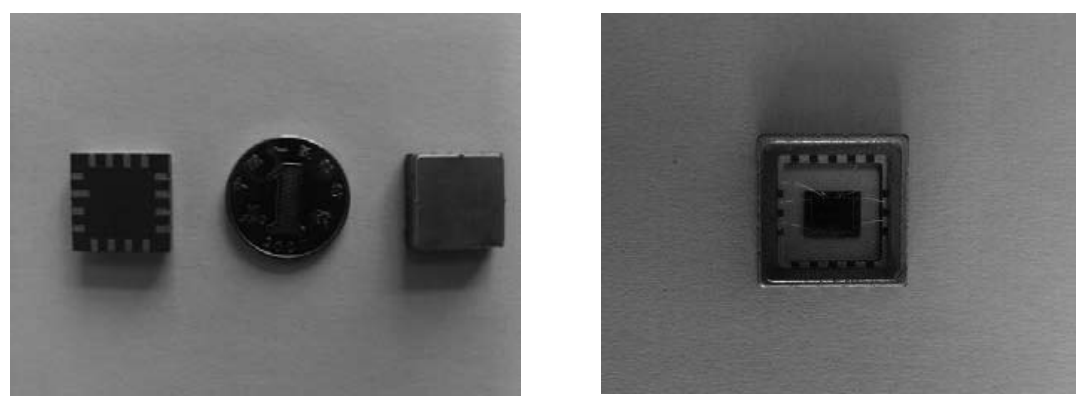

Fig. 14 (left). Packaged accelerometer.

Fig. 15 (right). Internal structure of the ceramic shell after wire bonding. 


\section{Measurement Results and Discussion}

An open-loop test of the accelerometer was performed on an M150 probe station. The test results show that the unloaded resonant frequencies of the unpackaged accelerometer resonators are 31.1 and $31.0 \mathrm{kHz}$. The relative errors between the test results and the design value are 0.5 and $0.8 \%$ respectively. Owing to the processing error, the unloaded resonant frequencies of the upper resonator and lower resonator are different.

The unloaded resonant frequencies of the packaged accelerometer resonators are 30.519 and $30.448 \mathrm{kHz}$, respectively, and the quality factor is 335 . Owing to the package stress, the resonant frequencies of the packaged accelerometer are lower than those of the unpackaged one.

The packaged accelerometer was fixed on an angular position platform. Different accelerations were applied to the accelerometers by changing the tilt angle of the platform. The frequencies of the two DETF resonators were measured and their difference was taken as the output of the accelerometer. Calculation of the scale factor of the accelerometer was carried out.

Figure 16 shows the test results of the scale factor of the packaged accelerometer. After fitting, the scale factor of the packaged accelerometer is $84.52 \mathrm{~Hz} / \mathrm{g}$.

\section{Conclusions}

The effect of the gap on the resonance characteristic of the resonant beams in a DETF structure was investigated through harmonic response analysis and modal analysis. The results show that the selection of the gap should take into account the tuning fork effect, the frequency difference between the operation mode and the interference modes, the processing error, and the damping. An improved microleverage mechanism was designed. Compared with the conventional mechanism, this microleverage mechanism can markedly improve the sensitivity of the accelerometer. On this basis, the design

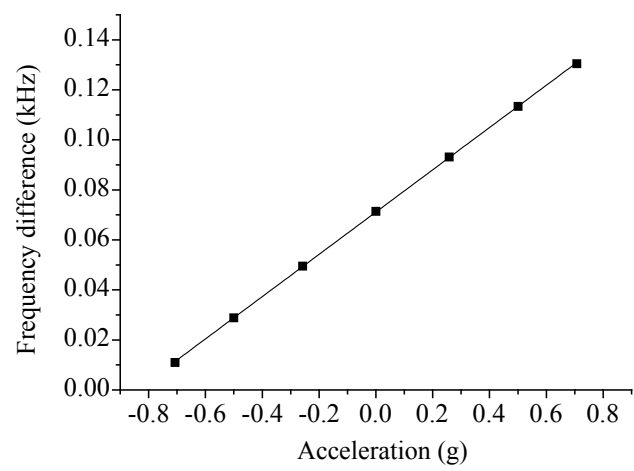

Fig. 16. Test results of the scale factor of the packaged accelerometer. 
of a micromechanical silicon resonant accelerometer with DETF resonators and single-stage microleverage mechanisms was accomplished. The accelerometer was fabricated using the SOG technique. According to the results of preliminary testing, the packaged accelerometer works well. The unloaded resonant frequencies of the packaged accelerometer resonators are 30.519 and $30.448 \mathrm{kHz}$, respectively. The quality factor is 335 and the scale factor is $84.52 \mathrm{~Hz} / \mathrm{g}$. This work is significant for the further development of micromechanical silicon resonant accelerometers.

\section{Acknowledgements}

This work was supported by the Foundation (No. KL201103) of Key Laboratory of Micro-Inertial Instrument and Advanced Navigation Technology, Ministry of Education, China; the National Natural Science Foundation of China (No. 61101021); the Jiangsu Provincial Natural Science Foundation of China (No. BK2010401); and the Guidance Foundation for Significant Research of Southeast University (No. 3222002105).

\section{References}

1 S. Y. Li, Z. L. Liu and X. Z. Wu: J. Natl. Univ. Defense Technol. 26 (2004) 34 (in Chinese).

2 J. Li, S. C. Fan, C. Li and Z. F. Yu: Transducer Microsyst. Technol. 30 (2011) 4 (in Chinese).

3 R. Hopkills, J. Miola and R. Setterlund: Draper Technol. Digest. 10 (2006) 4.

4 X. P. S. Xu and H. S. Yang: Struct. Multidiscip. Optim. 21 (2001) 246.

5 X. P. S. Xu, H. S. Yang and A. M. Agogino: IEEE Sens. J. 5 (2005) 1214.

6 S. K. Sung, J. G. Lee and T. Kang: Sens. Actuators, A 109 (2003) 1.

7 S. Seok, H. Kim and J. K. Chun: Proc. IEEE Sens. 2004 (IEEE, New York, 2004) p. 654.

8 P. A. Hassanpour, W. L. Cleghorn and E. Esmailzadeh: J. Sound Vib. 308 (2007) 287.

9 M. Aikele, K. Bauer and W. Ficker: Sens. Actuators, A 92 (2001) 161.

10 S. P. Beeby, G. Ensell and N. M. White: Eng. Sci. Educ. J. 9 (2000) 265.

11 S. P. Beeby, G. Ensell and B. R. Baker: J. Microelectromech. Syst. 9 (2000) 104.

12 C. X. Wan, D. D. Li, C. L. Li and X. Z. Chen: Navig. Contr. 9 (2010) 72 (in Chinese).

13 S. P. Beeby and M. J. Tudor: Micromech. Microeng. 5 (1995) 103.

14 M. H. Bao and H. Yang: Sens. Actuators, A 136 (2007) 3.

15 S. R. Wang, L. B. Huang and B. Yang: Micro Inertial Instruments and Micro Systems, ed. L. H. Lin (Weapon Industry Press, Beijing, 2011) Chap. 7 (in Chinese). 